\title{
AN EXISTENCE THEOREM FOR AN IMPLICIT INTEGRAL EQUATION WITH DISCONTINUOUS RIGHT-HAND SIDE
}

\author{
GIOVANNI ANELLO
}

Received 8 December 2004; Revised 9 March 2005; Accepted 22 March 2005

We establish a result concerning the existence of solutions for the following implicit integral equation: $g(u(t))=\varphi\left(t, x_{0}+\int_{0}^{t} f(\tau, u(\tau)) d \tau\right)$, where $\varphi$ is not supposed continuous with respect to the second variable.

Copyright (c) 2006 Giovanni Anello. This is an open access article distributed under the Creative Commons Attribution License, which permits unrestricted use, distribution, and reproduction in any medium, provided the original work is properly cited.

\section{Introduction}

Let $a>0, x_{0} \in \mathbb{R}$ and let $E$ be a metric space. Let $\left.f:[0, a] \times E \rightarrow\right] 0,+\infty[, g: E \rightarrow \mathbb{R}$ and $\varphi:[0, a] \times \mathbb{R} \rightarrow \mathbb{R}$ be given functions. The aim of this paper is to establish an existence theorem for an implicit integral equation of the type

$$
g(u(t))=\varphi\left(t, x_{0}+\int_{0}^{t} f(\tau, u(\tau)) d \tau\right)
$$

where function $\varphi$ is not supposed continuous with respect to the second variable. The reason for studying (1.1) arises mainly from the paper [3]. Indeed, [3, Theorem A] gives the existence of solutions for (1.1) assuming, among the other hypotheses, that $\varphi$ is a Carathéodory function and that $f$ does not depend on $t \in[0, a]$. We note that, using the arguments employed in the proof of Theorem A of [3], it seems that it is not possible neither to weaken the assumption of continuity of the function $\varphi$ in the second variable nor to assume $f$ dependent on $t \in[0, a]$. The purpose of the present paper goes just in this direction. Namely, studying (1.1) by means of quite different arguments from that ones used in [3], we are able to suppose $f$ dependent on $t \in[0, a]$ and to remove the continuity of $\varphi$ in the second variable. In particular, as regards to this latter, our assumptions allow $\varphi(t, \cdot)$ to be discontinuous at each point. The abstract framework where (1.1) is studied is that of set-valued analysis. In particular, we will deduce our result by using a recent selection theorem for multifunction of two variables (see [2, Theorem 2]) jointly to $[9$, Theorem 1]. 
2 Integral equations with discontinuous right-hand side

The reader who is interested to arguments related to the subject of the present paper is referred to [1] where singular integral equations and integral inclusions are studied.

\section{Basic definitions and notations}

Let $X, Y$ be two nonempty sets. A multifunction $F$ from $X$ into $Y$ is a function from $X$ into the family of all subsets of $Y$ and we briefly denote it by $F: X \rightarrow 2^{Y}$. The set $\operatorname{gr}(F):=$ $\{(x, y) \in X \times Y: y \in F(x)\}$ is called graph of $F$. For each $A \subseteq Y$, by $F^{-}(A)$ we denote the set $\{x \in X: F(x) \cap A \neq \varnothing\}$. We say that a function $f: X \rightarrow Y$ is a selection of $F$ if $f(x) \in$ $F(x)$ for all $x \in X$. If $X, Y$ are topological spaces, a multifunction $F: X \rightarrow 2^{Y}$ is said lower semicontinuous (briefly l.s.c.) at $x \in X$ if for any $y \in F(x)$ and any neighborhood $V$ of $y$ there exists a neighborhood $U$ of $x$ such that $F(z) \cap V \neq \varnothing$ for all $z \in U$. We recall that, when $F$ is a single-valued function, then lower semicontinuity coincides with the usual continuity. If $(X, \mathfrak{I})$ is a measurable space and $Y$ is a topological space, a multifunction $F: X \rightarrow 2^{Y}$ is said measurable when $F^{-}(A) \in \mathfrak{J}$ for any open set $A \subseteq Y$.

For all subset $A$ of a topological space, the symbol int $(A)$ stands for the interior of $A$. Also, for all subset $A$ of a normed space, the symbol $\overline{c o}(A)$ stands for the closed convex hull of $A$.

If $X$ is a topological space, we denote by $\mathscr{H}(X)$ the Borel $\sigma$-algebra of $X$. If $\mu$ is a positive regular Borel measures on $X$, we denote by $\mathscr{T}_{\mu}(X)$ the completion of the Borel $\sigma$-algebra of $X$ with respect to $\mu$.

A Polish space is a topological space $X$ which is separable and metrizable by a complete metric.

Finally, if $(X, d)$ is a metric space, we put $B(x, r)=\{y \in X: d(x, y)<r\}$ for all $r>0$ and $x \in X$.

We close this section stating, for the reader's convenience, the following results (the first two already quoted in the introduction) which will be used in the proof of our main result.

Theorem 2.1 (see [2, Theorem 2]). Let T, X be two Polish spaces and let $\mu, \psi$ be two positive regular Borel measures on $T$ and $X$, respectively, with $\mu$ finite and $\psi \sigma$-finite. Let $S$ be a separable metric space, $F: T \times X \rightarrow 2^{S}$ a multifunction with non empty complete values, and let $E \subseteq X$ be a given set. Finally, let assume that:

(i) $F$ is $\mathscr{T}_{\mu}(T) \otimes \mathscr{B}(X)$-measurable;

(ii) for a.a. $t \in T$, one has that $\{x \in X: F(t, \cdot)$ is not lower semicontinuous at $x\} \subseteq E$.

Then, there exists a selection $\phi: T \times X \rightarrow S$ of $F$ and a negligible set $R \subseteq X$ such that

(i) $)^{\prime} \phi(\cdot, x)$ is $\mathscr{T}_{\mu}(T)$-measurable for each $x \in X \backslash(E \cup R)$;

(ii)' for a.a. $t \in T$, one has that $\{x \in X: \phi(t, \cdot)$ is not continuous at $x\} \subseteq E \cup R$.

Theorem 2.2 (see [9, Theorem 1]). Let $(T, \mathfrak{J}, \mu)$ be a finite non-atomic complete measure space; $V$ a non-empty set; $\left(X,\|\cdot\|_{X}\right),\left(Y,\|\cdot\|_{Y}\right)$ two separable real Banach spaces, with $Y$ finite-dimensional; $p, q, s \in[1,+\infty]$, with $q<+\infty$ and $q \leq p \leq s ; \Psi: V \rightarrow L^{s}(T, Y) a$ surjective and one-to-one operator; $\Phi: V \rightarrow L^{1}(T, X)$ an operator such that, for every $v \in$ $L^{s}(T, Y)$ and every sequence $\left\{v_{n}\right\}$ in $L^{s}(T, Y)$ weakly converging to $v$ in $L^{q}(T, Y)$, the sequence $\left\{\Phi\left(\Psi^{-1}\left(v_{n}\right)\right)\right\}$ converges strongly to $\left\{\Phi\left(\Psi^{-1}(v)\right)\right\}$ in $L^{1}(T, X) ; \chi:[0,+\infty[\rightarrow[0,+\infty]$ 
a non-decreasing function such that

$$
\underset{t \in T}{\operatorname{ess} \sup }\|\Phi(u)(t)\|_{X} \leq \chi\left(\|\Psi(u)\|_{L^{p}(T, Y)}\right)
$$

for all $u \in V$.

Further, let $F: T \times X \rightarrow 2^{Y}$ be a multifunction, with non-empty closed convex values, satisfying the following conditions:

(i) for $\mu$-almost every $t \in T$, the multifunction $F(t, \cdot)$ has closed graph;

(ii) the set $\{x \in X$ : the multifunction $F(\cdot, x)$ is $\mathfrak{I}$ - measurable $\}$ is dense in $X$;

(iii) there exists a number $r>0$ such that the function $t \rightarrow \sup _{\|x\|_{X} \leq \chi(r)} d\left(\vartheta_{Y}, F(t, x)\right)$ belongs to $L^{s}(T)$ and its norm in $L^{p}(T)$ is less or equal to $r$.

Under such hypotheses, there exists $\tilde{u} \in V$ such that

$$
\begin{gathered}
\Psi(\tilde{u})(t) \in F(t, \Phi(\tilde{u})(t)) \quad \mu-\text { a.e. in } T, \\
\|\Psi(\tilde{u})(t)\|_{Y} \leq \sup _{\|x\|_{X} \leq \chi(r)} d\left(\vartheta_{Y}, F(t, x)\right) \quad \mu-\text { a.e. in } T .
\end{gathered}
$$

Theorem 2.3 (see [8, Theorem 2.4]). Let $\Sigma$ be a connected and locally connected topological space, $I$ a real interval with extremes $a, b$ and $f: \Sigma \rightarrow I$ a continuous function such that $f^{-1}(t)=\varnothing$ for all $\left.t \in\right] a, b\left[\right.$. Then, there exists a set $\Sigma^{*} \subseteq \Sigma$ such that

(i) the set $f^{-1}(t) \cap \Sigma^{*}$ is non-empty and closed for all $t \in I$;

(ii) the function $f_{\mid \Sigma^{*}}$ is open.

Theorem 2.4 (see [5, Proposition 2]). Let $I \subset \mathbb{R}$ be an interval, $\psi: I \times \mathbb{R}^{n} \rightarrow \mathbb{R}^{n}$ a given function and $D$ a countable and dense subset of $\mathbb{R}^{n}$. Assume that:

(i) for each $t \in I$, the function $\psi(t, \cdot)$ is bounded;

(ii) for each $x \in D$, the function $\psi(\cdot, x)$ is measurable.

Let $H: I \times \mathbb{R}^{n} \rightarrow \mathbb{R}^{n}$ be the multifunction defined by

$$
H(t, x)=\bigcap_{m \in \mathbb{N}} \overline{\overline{c o}} \overline{\left(\bigcup_{y \in P,|y-x| \leq 1 / m}\{\psi(t, y)\}\right)} .
$$

Then, one has:

(a) $H$ has nonempty closed convex values;

(b) for all $x \in \mathbb{R}$, the multifunction $H(\cdot, x)$ is measurable;

(c) for each $t \in I$, the multifunction $H(t, \cdot)$ has closed graph;

(d) if $t \in T$ and $\psi(t, \cdot)$ is continuous at $x \in \mathbb{R}^{n}$, then $H(t, x)=\{\psi(t, x)\}$.

\section{Main result}

Before proving our main result, we need the following two well known lemmas. We give their proofs for sake of clearness.

Lemma 3.1. Let $(T, \mathfrak{I})$ be a measurable space, $X$ be a separable metric space and $Y$ a topological space. Let $F: T \times X \rightarrow 2^{Y}$ be a given multifunction. Assume that

(a) $F(t, \cdot)$ is l.s.c for all $t \in T$; 
4 Integral equations with discontinuous right-hand side

(b) there exists a countable dense subset $D$ of $X$ such that $F(\cdot, x)$ is measurable for all $x \in D$.

Then, $F$ is $\mathfrak{I} \otimes \mathscr{B}(X)$-measurable.

Proof. Let $\Omega$ be an open subset of $Y$. It is easily checked that the following equality

$$
F^{-}(\Omega)=\bigcap_{k \in \mathbb{N}} \bigcup_{x \in D}\{t \in T: F(t, x) \cap \Omega\} \times B(x, 1 / k)
$$

holds. Thus, by assumption (a) and (b) one has $F^{-}(\Omega) \in \mathfrak{J} \otimes \mathscr{B}(X)$ and conclusion follows.

Lemma 3.2. Let $(T, \mathfrak{I}, \mu)$ be a complete finite measure space, $X$ be a Polish space, $Y, Z$ be two topological spaces, $F: T \times X \rightarrow Z$ and $H: T \times Y \rightarrow 2^{X}$ be two multifunctions. Assume that

(a) $F$ is $\mathfrak{I} \otimes \mathscr{B}(X)$-measurable,

(b) $H$ is $\mathfrak{I} \otimes \mathscr{B}(Y)$-measurable and has closed values.

Then, the multifunction $G$ defined by $G(t, y)=F(t, H(t, y))$ for all $(t, y) \in T \times Y$ is $\mathfrak{I} \otimes$ $\mathscr{B}(Y)$-measurable.

Proof. Let $\Omega$ be an open subset of $Z$. Then, $F^{-}(\Omega)$ is $\mathfrak{I} \otimes \mathscr{B}(X)$-measurable. Hence, the set

$$
A=\left\{(t, y, x) \in T \times Y \times X:(t, x) \in F^{-}(\Omega)\right\}
$$

is $\mathfrak{I} \otimes \mathscr{B}(Y) \otimes \mathscr{B}(X)$-measurable. Moreover, owing to [7, Theorem 3.5], $\operatorname{gr}(H)$ is $\mathfrak{I} \otimes$ $\mathscr{B}(Y) \otimes \mathscr{B}(X)$-measurable as well and, consequently, so is the set $A \cap \operatorname{gr}(H)$. Now, it is easily seen that

$$
G^{-}(\Omega)=P_{T \times Y}(\operatorname{gr}(H) \cap A),
$$

where $P_{T \times Y}$ denotes the projection on $T \times Y$. Thus, by [4, Theorem III.23], one has $G^{-}(\Omega) \in \mathfrak{J} \otimes \mathscr{B}(Y)$ from which the conclusion follows.

Now, we state and prove the main result. In the sequel we will denote by $\mathscr{L}([0, a])$ the Lebesgue $\sigma$-algebra of $[0, a]$ and measurability, unless explicitly specified, will be understood with respect to this latter. Also, we denote by $m$ the Lebesgue-measure on $\mathscr{L}([0, a])$.

TheOREm 3.3. Let E be a compact connected and locally connected metric space and $x_{0} \in \mathbb{R}$. Let $f:[0, a] \times E \rightarrow \mathbb{R}, g: E \rightarrow \mathbb{R}$ and $\varphi:[0, a] \times \mathbb{R} \rightarrow \mathbb{R}$ be given functions. Assume that there exists a function $\varphi_{1}:[0, a] \times \mathbb{R} \rightarrow \mathbb{R}$ such that

(i) there exist $S, S_{1} \subseteq \mathbb{R}$ with $m(S)=m\left(S_{1}\right)=0$ and $S_{1}$ closed, such that $\left\{x \in \mathbb{R}: \varphi_{1}(t, \cdot)\right.$ is discontinuous at $x\} \subseteq S_{1}$ and $\left\{x \in \mathbb{R}: \varphi_{1}(t, x) \neq \varphi(t, x)\right\} \subseteq S$ for a.a. $t \in[0, a]$;

(ii) $\varphi_{1}(\cdot, x)$ is measurable for a.a. $x \in \mathbb{R}$;

(iii) $\varphi_{1}\left(\{t\} \times\left(\mathbb{R} \backslash S_{1}\right)\right) \subseteq g(E)$ for a.a. $t \in[0, a]$. Moreover, assume that

(iv) $g$ is continuous and $\operatorname{int}\left(g^{-1}(r)\right)=\varnothing$ for all $r \in \operatorname{int}(g(E))$;

(v) $f(t, \cdot)$ is continuous for a.a. $t \in[0, a]$ and $f(\cdot, z)$ is measurable for all $z$ belonging to a countable dense subset of $E$; 
(vi) there exist $\alpha:[0, a] \rightarrow] 0,+\infty\left[\right.$ and $\beta \in L^{1}([0, a])$ such that $\alpha(t) \leq f(t, z) \leq \beta(t)$ for a.a. $t \in[0, a]$ and $z \in g^{-1}\left(\varphi_{1}\left(\{t\} \times\left(\mathbb{R} \backslash S_{1}\right)\right)\right)$.

Then, there exists a measurable function $u:[0, a] \rightarrow E$ which solves (1.1).

Proof. Without loss of generality, we can suppose that conditions (i), (iii), (v) and (vi) hold for all $t \in[0, a]$. Since $E$ is a compact metric space, then $E$ is separable. Hence, in particular, $E$ is a Polish space. By condition (ii), we can find a countable set $P \subseteq \mathbb{R} \backslash S_{1}$ dense in $\mathbb{R}$ such that

$$
\varphi_{1}(\cdot, x) \text { is measurable } \forall x \in P .
$$

Moreover, taking into account of (iv) and hypotheses on $E$, we can apply Theorem 2.3. Therefore, there exists a set $Y \subseteq\left[a,+\infty\right.$ [ such that $g^{-1}(\sigma) \cap Y$ is nonempty and closed in $E$ (hence compact because $E$ is like) for each $\sigma \in g(E)$ and the multifunction $g^{-1}(\cdot) \cap Y$ is l.s.c. in $g(E)$. Now, fix $\bar{x} \in P$ and put

$$
\hat{\varphi}(t, x)= \begin{cases}\varphi_{1}(t, x) & \text { if }(t, x) \in[0, a] \times\left(\mathbb{R} \backslash S_{1}\right), \\ \varphi_{1}(t, \bar{x}) & \text { if }(t, x) \in[0, a] \times S_{1} .\end{cases}
$$

By Lemma 3.1 we have that $\hat{\varphi}$ is $\mathscr{L}([0, a]) \otimes \mathscr{B}(\mathbb{R})$-measurable. Further, being $S_{1}$ closed, one has

$$
\{x \in \mathbb{R}: \hat{\varphi}(t, \cdot) \text { is discontinuous at } x\} \subseteq S_{1}
$$

for a.a. $t \in[0, a]$. At this point, we put

$$
F(t, x)=f\left(t, g^{-1}(\hat{\varphi}(t, x)) \cap Y\right)
$$

for all $(t, x) \in[0, a] \times \mathbb{R}$. From the definition of $\hat{\varphi}$ and condition (iii) $F$ has nonempty values. Being $g^{-1}(\hat{\varphi}(t, x)) \cap Y$ compact and $f(t, \cdot)$ continuous for all $t \in[0, a]$ and $x \in \mathbb{R}$, we also have that $F$ has, in particular, closed values in $\mathbb{R}$ (actually, these latter are compact as well). Moreover, observe that

$$
\{x \in \mathbb{R}: F(t, \cdot) \text { is not 1.s.c. at } x\} \subseteq S_{1} .
$$

Now, condition (v) and Lemma 3.1 imply that $f$ is $\mathscr{L}([0, a]) \otimes \mathscr{B}(E)$-measurable. So, by Lemma 3.2, we have that $F$ is $\mathscr{L}([0, a]) \otimes \mathscr{B}(E)$-measurable. Therefore, we can apply Theorem 2.1. Then, there exist a selection $\psi$ of $F$ and a set $D \subset \mathbb{R}$ having measure 0 such that

$$
\begin{gathered}
\{x \in \mathbb{R}: \psi(t, \cdot) \text { is discontinuous at } x\} \subseteq S_{1} \cup D, \\
\psi(\cdot, x) \text { is measurable } \forall x \in \mathbb{R} \backslash\left(S_{1} \cup D\right) .
\end{gathered}
$$

Hence, $\psi(t, \cdot)$ turns out bounded for all $t \in[0, a]$. Consequently, the multifunction $H$ : $[0, a] \times \mathbb{R} \rightarrow 2^{\mathbb{R}}$ defined by setting

$$
H(t, x)=\bigcap_{m \in \mathbb{N}} \overline{\overline{\operatorname{co}}} \overline{\left(\bigcup_{y \in P,|y-x| \leq 1 / m}\{\psi(t, y)\}\right)}
$$


6 Integral equations with discontinuous right-hand side

satisfies properties (a), (b), (c), (d) of Theorem 2.4. In particular one has $H(t, x)=$ $\{\psi(t, x)\}$ for a.a. $t \in I$ and all $x \in \mathbb{R} \backslash S_{1} \cup D$. Moreover, by the above construction, it follows that

$$
H(t, x) \subseteq[\alpha(t), \beta(t)] \quad \forall x \in \mathbb{R}, t \in[0, a] .
$$

Now, we want to apply Theorem 2.2 to the multifunction $H$, taking $T=[0, a], X=Y=$ $\mathbb{R}, s=q=p=1, V=L^{1}([0, a]), \Psi(u)=u, \Phi(u)(t)=x_{0}+\int_{a}^{t} u(\tau) d \tau, \chi \equiv+\infty$ and $r=$ $\int_{0}^{a}|\beta(t)| d t$. To this aim, we observe the following facts

(j) $\Phi\left(L^{1}([0, a])\right) \subseteq A C([0, a])$, where $A C([0, a])$ is the set of all absolutely continuous function on $[0, a]$;

(jj) let $\left\{v_{n}\right\}$ be a sequence in $L^{1}([0, a])$ weakly converging to $v \in L^{1}([0, a])$. Then, being $\Phi$ affine, one has that $\Phi\left(v_{n}\right)$ is pointwise converging in $[0, a]$. Since, in particular, $\left\{v_{n}\right\}$ bounded in $L^{1}([0, a])$, we easily deduce that $\left|\Phi\left(v_{n}\right)(t)\right| \leq \sup _{n \in \mathbb{N}} \int_{0}^{a}\left|v_{n}(\tau)\right| d \tau+\left|x_{0}\right|<+\infty$ for a.a. $t \in[0, a]$. Hence, applying the dominated convergence theorem, we have that $\left\{\Phi\left(v_{n}\right)\right\}$ converges strongly in $L^{1}([0, a])$;

(jij) the function $t \in[0, a] \rightarrow \sup _{x \in \mathbb{R}}|H(t, x)|$ is measurable (see, for instance, [9, page $262])$ and, by (3.11), it belongs to $L^{1}([0, a])$ and its norm in this space is less or equal to $\int_{0}^{a}|\beta(t)| d t$.

Consequently, all the assumptions of Theorem 2.2 are fulfilled. Hence, there exists $v_{0} \in$ $L^{1}([0, a])$ such that

$$
v_{0}(t) \in H\left(t, x_{0}+\int_{0}^{t} v_{0}(\tau) d \tau\right) \quad \text { for a.a. } t \in[0, a]
$$

Put $u_{0}(t)=x_{0}+\int_{0}^{t} v_{0}(\tau) d \tau$ for every $t \in[0, a]$. By (3.11) and since $\alpha(t)>0$ for all $t \in$ $[0, a]$, we have $u_{0}^{\prime}(t)>0$ for a.a. $t \in[0, a]$. So, by [10, Theorem 2], the function $u_{0}^{-1}$ is absolutely continuous. Thus, by [6, Theorem 18.25], the set $\Sigma=u_{0}^{-1}\left(S \cup S_{1} \cup D\right)$ has measure 0 . Now, if $t \in[0, a] \backslash \Sigma$, one has $u_{0}(t) \in \mathbb{R} \backslash\left(S \cup S_{1} \cup D\right)$. Hence, by property (d) of multifunction $H$, by (3.12), and taking into account of the construction of $\hat{\varphi}$, it turns out

$$
u_{0}^{\prime}(t) \in f\left(t, g^{-1}\left(\varphi\left(t, u_{0}(t)\right)\right)\right) \quad \text { for a.a. } t \in[0, a] .
$$

At this point, we put

$$
\Gamma(t)=f(t, \cdot)^{-1}\left(u_{0}^{\prime}(t)\right) \cap g^{-1}\left(\varphi\left(t, u_{0}(t)\right)\right)
$$

for all $t \in[0, a]$. Then, $\Gamma$ has closed values and, by (3.13), they are non empty. Now, observe that the sets

$$
\begin{gathered}
\left\{(t, x) \in[0, a] \times E: f(t, x)=u_{0}^{\prime}(t)\right\}, \\
\left\{(t, x) \in[0, a] \times E: g(x)=\varphi\left(t, u_{0}(t)\right)\right\}
\end{gathered}
$$

are $\mathscr{L}([0, a]) \otimes \mathscr{B}(E)$-measurable. Since these latter are the graphs of the multifunctions

$$
\begin{aligned}
& t \in[0, a] \longrightarrow f(t, \cdot)^{-1}\left(u_{0}^{\prime}(t)\right), \\
& t \in[0, a] \longrightarrow g^{-1}\left(\varphi\left(t, u_{0}(t)\right)\right),
\end{aligned}
$$


respectively, then by [7, Theorem 3.5 and Corollary 4.2], we have that the multifunction $\Gamma$ is measurable. Hence, by Kuratowski and Ryll-Nardzewski theorem, there exists a measurable function $u:[0, a] \rightarrow E$ such that $u(t) \in \Gamma(t)$ for a.a. $t \in[0, a]$. In particular, by (3.13), we have $f(t, u(t))=u_{0}^{\prime}(t)$ and $g(u(t))=\varphi\left(t, u_{0}(t)\right)$ for a.a. $t \in[0, a]$. From this we deduce that

$$
g(u(t))=\varphi\left(t, x_{0}+\int_{0}^{t} f(\tau, u(\tau)) d \tau\right) \quad \text { for a.a. } t \in[0, a] .
$$

So, the proof is complete.

Remark 3.4. The compactness of the metric space $E$ is used in the proof of Theorem 3.3 in order that $F$ has closed values. Nevertheless, if $E$ is a connected and locally connected Polish space only, we can get that $F$ has closed values assuming, in addiction, that $f(t, \cdot)$ is a closed function for a.a. $t \in[0, a]$, namely having the following property: $f(t, C)$ is a closed set in $E$ for all closed set $C$ in $\mathbb{R}$ and for a.a. $t \in[0, a]$.

Example 3.5. We present a simple example of application of Theorem 3.3 where the function $\varphi$ is discontinuous at each point with respect to the second variable:

let $E=\left\{x \in \mathbb{R}^{n}:\|x\|_{n} \leq 1\right\}$ be the unit ball of $\mathbb{R}^{n} ; x_{0}=0$ and $a=1$. Define $g(x)=$ $\sin \left(\pi\|x\|_{n}\right)$ for all $x \in E$. It is immediate to check that $g(E)=[0,1]$ and that $\operatorname{int}\left(g^{-1}(r)\right)=$ $\varnothing$ for all $r \in[0,1]$. Also define $\varphi(t, x)=\alpha(t) \chi_{\mathbb{R} \backslash \mathbb{Q}}(x)$ where $\alpha$ is a measurable function with $\alpha(t) \in] 0,1]$ for a.a. $t \in[0,1]$ and $\chi_{\mathbb{R} \backslash \mathbb{Q}}$ is the characteristic function of $\mathbb{R} \backslash \mathbb{Q}: \chi_{\mathbb{R} \backslash \mathbb{Q}}(x)$ $=1$ if $x \in \mathbb{R} \backslash \mathbb{Q}$ and $\chi_{\mathbb{R} \backslash \mathbb{Q}}(x)=0$ if $x \in \mathbb{Q}$. Note that for a.a. $t \in[0,1], \varphi(t, \cdot)$ is discontinuous at each point of $\mathbb{R}$. With this choice of $\varphi$ we see that conditions (i), (ii), (iii) are satisfied if we take $\varphi_{1}(t, x)=\alpha(t)$ for all $(t, x) \in[0,1] \times \mathbb{R}$. So, applying Theorem 3.3, we have that for every Carathéodory function $f:[0,1] \times E \rightarrow] 0,+\infty\left[\operatorname{such}_{\text {that }} \sup _{x \in E} f(\cdot, x)\right.$ $\in L^{1}([0,1])$, there exists $u \in L^{\infty}([0,1])$ such that

$$
\sin \left(\pi\|u(t)\|_{n}\right)=\alpha(t) \chi_{\mathbb{R} \backslash \mathbb{Q}}\left(\int_{0}^{t} f(\tau, u(\tau)) d t\right) \quad \text { for a.a. } t \in[0,1] .
$$

\section{References}

[1] R. P. Agarwal, M. Meehan, and D. O'Regan, Nonlinear Integral Equations and Inclusions, NOVA, New York, 2001.

[2] G. Anello and P. Cubiotti, Parametrization of Riemann-measurable selections for multifunctions of two variables with application to differential inclusions, Annales Polonici Mathematici 83 (2004), no. $2,179-187$.

[3] G. Bonanno, Differential inclusions with nonconvex right hand side and applications to implicit integral and differential equations, Rendiconti Accademia Nazionale delle Scienze detta dei XL Memorie di Matematica e Applicazioni Serie V 20 (1996), no. 1, 193-203.

[4] C. Castaing and M. Valadier, Convex Analysis and Measurable Multifunctions, Lecture Notes in Mathematics, vol. 580, Springer, Berlin, 1977.

[5] P. Cubiotti, Non-autonomous vector integral equations with discontinuous right-hand side, Commentationes Mathematicae Universitatis Carolinae 42 (2001), no. 2, 319-329.

[6] E. Hewitt and K. Stromberg, Real and Abstract Analysis. A Modern Treatment of the Theory of Functions of a Real Variable, Springer, New York, 1965.

[7] C. J. Himmelberg, Measurable relations, Fundamenta Mathematicae 87 (1975), 53-72. 
8 Integral equations with discontinuous right-hand side

[8] B. Ricceri, Sur la semi-continuité inférieure de certaines multifonctions [On the lower semicontinuity of certain multifunctions], Comptes Rendus de l'Académie des Séances. Série I Mathématique 294 (1982), no. 7, 265-267 (French).

[9] O. N. Ricceri and B. Ricceri, An existence theorem for inclusions of the type $\Psi(u)(t) \in F(t, \Phi(u)(t))$ and application to a multivalued boundary value problem, Applicable Analysis 38 (1990), no. 4, 259-270.

[10] A. Villani, On Lusin's condition for the inverse function, Rendiconti del Circolo Matematico di Palermo. Serie II 33 (1984), no. 3, 331-335.

Giovanni Anello: Department of Mathematics, University of Messina, 98166 Sant' Agata,

Messina, Italy

E-mail address: anello@dipmat.unime.it 